\section{Uncommon first diagnosis of metastatic papillary thyroid carcinoma with "signet-ring" cells morphology through pericardial effusion}

\author{
Angeliki Cheva1, Sokratis Tsagkaropoulos ${ }^{2 *}$, Panagiotis Pepis², \\ Antonia Syrnioti ${ }^{1}$ and Christoforos Foroulis ${ }^{2}$
}

1Department of Pathology, School of Medicine, Aristotle University of Thessaloniki, Greece ${ }^{2}$ Department of Cardiothoracic Surgery, AHEPA University Hospital, Thessaloniki, Greece

\section{Abstract}

Malignant pericardial effusion is a usual and crucial exacerbation in malignancies. In some cases, pericardial effusion may be the first manifestation of the disease. Neoplastic pericarditis is extremely rare in thyroid cancer. Hereby, we describe the case of a 54-year-old man submitted to the hospital for chest pain and dyspnea. Clinical and radiological examinations were suggestive of pericardial effusion and mediastinal lymphadenopathy. The patient underwent pericardial drainage and surgical biopsy of a paratracheal lymph node. Pericardial biopsies were also taken. Histology study was compatible with metastatic papillary thyroid carcinoma with "signet-ring" cell morphology. Extended study of thyroid gland and function was performed and he was referred to oncologists for further treatment.

Clinical presentation and histology are discussed emphasizing the rare correlation between pericardial pathology and metastatic thyroid carcinomas as the first diagnosis. Histopathologic findings of "signet-ring" cell morphology are very challenging for a differential diagnosis between thyroid carcinoma and other metastatic adenocarcinomas.

\section{More Information}

*Address for Correspondence:

Sokratis Tsagkaropoulos, St. Kiriakidi 1, 54636,

Thessaloniki, Greece,

Email: stsagkarop@yahoo.com

Submitted: October 28, 2021

Approved: January 19, 2022

Published: January 20, 2022

How to cite this article: Cheva A,

Tsagkaropoulos S, Pepis P, Syrnioti A, Foroulis C. Uncommon first diagnosis of metastatic papillary thyroid carcinoma with "signet-ring" cells morphology through pericardial effusion. Arch Case Rep. 2022; 6: 005-008.

DOI: 10.29328/journal.acr.1001057

Copyright License: (c) 2022 Cheva A, et al. This is an open access article distributed under the Creative Commons Attribution License, which permits unrestricted use, distribution, and reproduction in any medium, provided the original work is properly cited.

Keywords: Papillary thyroid carcinoma; Pericardial effusion; Metastatic adenocarcinoma; Signet ring cell thyroid carcinoma; Lymphadenopathy

(W) Check for updates

OPEN ACCEss

\section{Case presentation}

A 54-year-old man with a history of arterial hypertension was referred to cardiologists because of weakness, chest pain and dyspnea. Electrocardiogram detected sinus rhythm and routine blood tests revealed no leukocytosis and normal cardiac markers. Acute coronary disease was excluded. Echocardiography revealed pericardial effusion of more than $2 \mathrm{~cm}$ around the heart and presence of swinging heart with normal blood pressure. Urgent echo-guided pericardiocentesis was performed for both therapeutic and diagnostic purposes and about $800 \mathrm{ml}$ of serosanguinous fluid was drained resolving symptomatology. Cytology of the pericardial fluid was positive for malignant cells and consistent with metastatic adenocarcinoma. The patient was examined further in order to identify the primary site of malignancy. A whole body computed tomography (CT) scan followed by a positron emission tomography (PET) scan showed extended mediastinal and abdominal lymphadenopathy with high metabolic activity (SUVmax 10.2), millimetric pulmonary nodules with mild metabolic activity (SUVmax between 3 and 3.8) and remaining pericardial effusion (Figure 1A,B). Thus, he was transferred to the thoracic surgery department for evaluation. It was decided to perform a subxiphoid pericardial window, for definitive treatment of the effusion, as well as a pericardial biopsy. Furthermore, a paratracheal lymph node was biopsied through cervical video-mediastinoscopy under general anesthesia in order to obtain enough tissue for histology. A sample of about $2 \times 2 \mathrm{~cm}$ pericardium tissue, as well as an unexpected finding of solid nodule in the abdominal fat tissue were sent for histopathologic examination. Furthermore, in the same setting, samples of pretracheal and right lower paratracheal lymph nodes were also sent for an exam (Figure 1C,D).

The histological examination of all the samples revealed 
large clusters of neoplastic cells in a loose tissue background or in lymph node tissue. They varied in size from intermediate to large, with a high nuclear/cytoplasmic ratio, high nuclear atypia and increased mitotic activity. The majority of the tumor cells had prominent cytoplasmic vacuoles, which pushed the nuclei to the periphery of the cells and developed a morphology of "signet ring" cells (Figure 2A,B). Some had round "empty" nuclei with grooving membrane and small inconspicuous nucleoli. Follicular differentiation was rare (Figure 2C).

In differential diagnosis, the signet ring morphology of neoplastic cells initially indicates a metastatic signet ring cell carcinoma:

A primary lung cancer could be possible, due to pericarditis and the existence of malignant cells in the pericardial fluid.

The solid nodule in the abdominal fat also renders possible the case of a signet ring cell carcinoma of the stomach or of the other side of the gastrointestinal tract.

In addition, because of the "empty" nuclei with grooving membrane, we cannot exclude a metastatic papillary thyroid carcinoma. Furthermore, after extensive and detailed examination of all tissue biopsies, the occurrence of rare, small glands could indicate the follicular variant of the papillary thyroid carcinoma.

The following immunohistochemical stains were performed: Cytokeratin 7, TTF1, NapsinA, Cytokeratin 20, CDX2, Thyroglobulin, Galectin-3 and PSA. We repeated the stain process on two different paraffin blocks, which consisted of pericardial tissue as well as lymph node tissue. All the neoplastic cells were strongly positive for CK7, TTF1, Thyroglobulin and Galectin-3 (Figure 3). In contrast, they were negative for NapsinA, CDX2, CK20 and PSA. Due to prominent cytoplasmic vacuoles, the histochemical stain of periodic acidschiff (PAS) was also performed. It was positive, revealing the presence of mucin (Figure 2D).

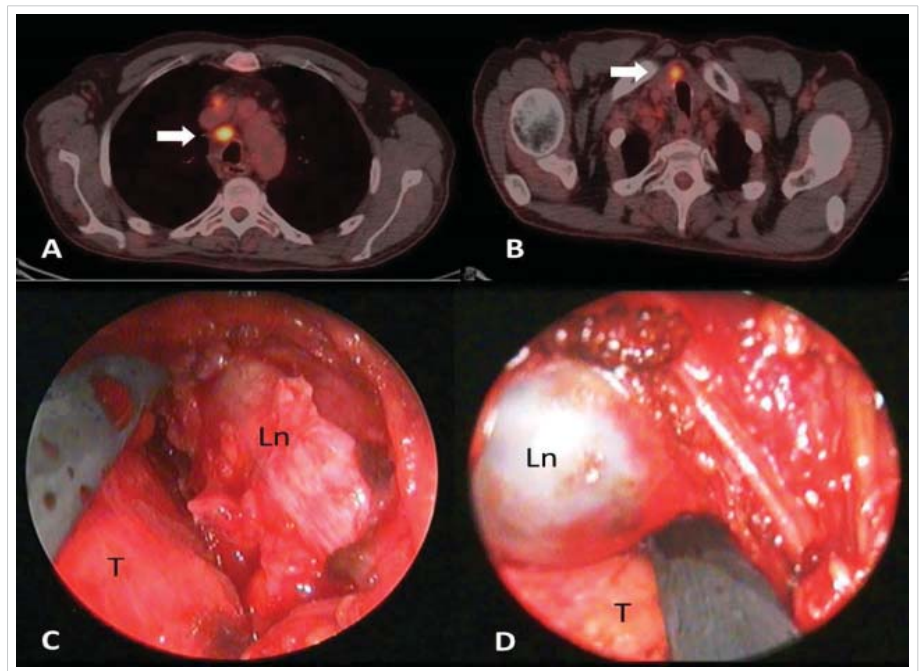

Figure 1: PET/CT scan revealing high metabolic activity in right paratracheal $(\mathrm{A})$ and pretracheal $(B)$ lymph nodes. Introperative findings corresponding to the PET/ CT scan images (C, D). LN: lymph nodes, T: trachea.
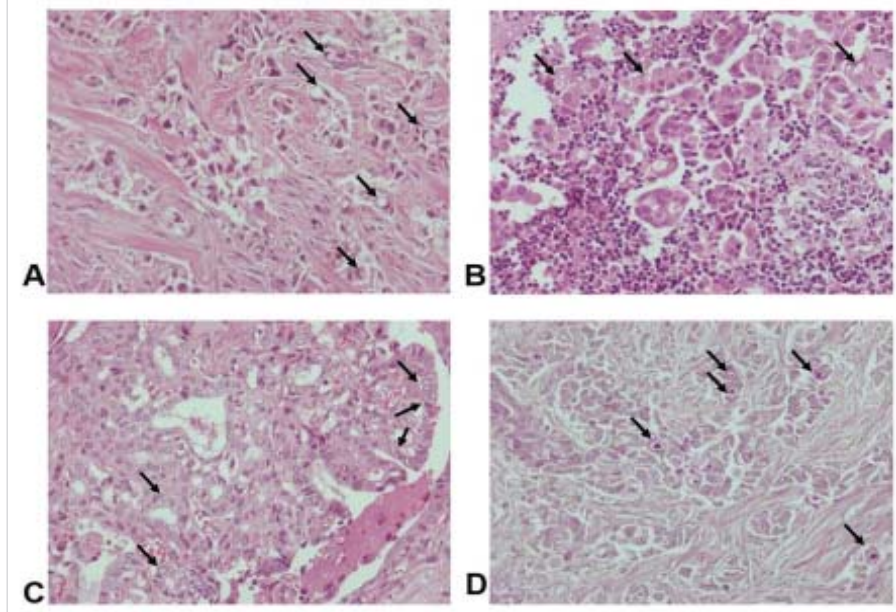

Figure 2: A, B. Hematoxylin and eosin x200: "Signet-ring" cell morphology of neoplastic cells in pericardial tissue (A) and lymph node (B), C. "Empty" nuclei with grooving membrane and small inconspicuous nucleoli with papillary and follicular growth pattern in pericardial tissue, D. Periodic Acid-Schiff (PAS) $\times 200$, revealing the presence of intracytoplasmic music. Arrows indicate pathologic cells.

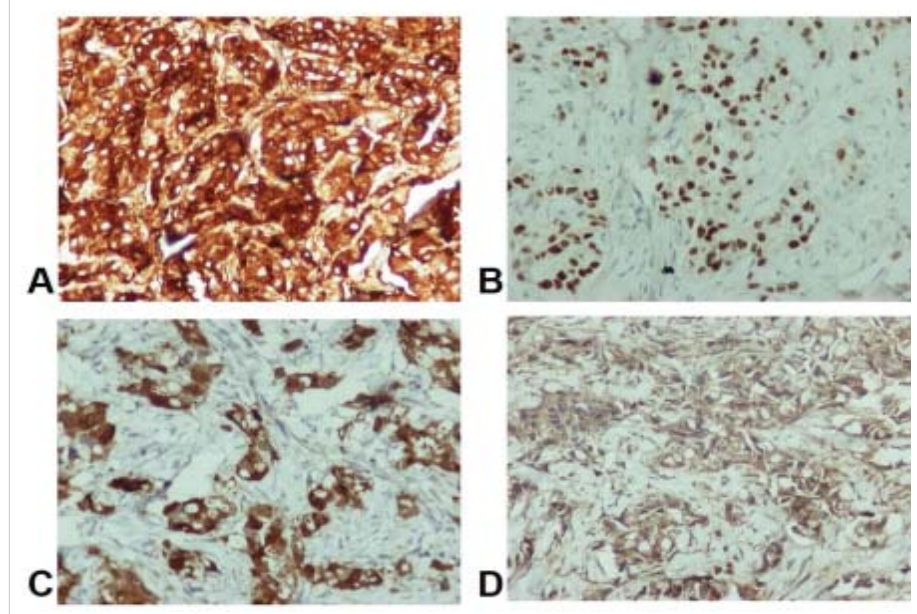

Figure 3: Positive expression of neoplastic cells in immunohistochemical stains: A. CK7 $\times 200$ (pericardial tissue), B. TTF1 x200 (lymph node), C. Thyroglobulin $\times 200$ (pericardial tissue), D. Galectin $-3 \times 200$ (pericardial tissue).

Despite the unexpected morphology of the neoplastic cells and according to the current immunophenotype, our diagnosis was compatible with metastatic papillary thyroid carcinoma with "signet-ring" cell (SRC) morphology [1], stage IV according to the WHO classification system. Careful revision of the radiologic images was made to find out a missing thyroid pathology with negative result. Thyroid hormones were also in normal range. The patient was referred to expertized oncologists in thyroid cancer for detail exam of thyroid gland and medical treatment. Surgery was excluded based on the advanced stage of the neoplasm.

\section{Discussion}

There are different etiological factors regarding cancer which might lead to pericardial effusion, such as solid masses as well as blood cancer, whereas primary neoplasms of the pericardium are less frequent. In some cases, pericardial effusion may be the first manifestation of the disease, and that is why malignancy must be excluded in every case of an acute 
pericardial disease with cardiac tamponade at presentation, rapidly increasing pericardial effusion and an incessant or recurrent course [2]. Thus, the definite differentiation of malignant pericardial effusion and rapid diagnosis are of particular therapeutic and prognostic importance.

Histological examination of the pericardial biopsy or cytological examination of the pericardial fluid is key point to a patient's diagnosis. Neoplastic pericarditis maintains only a small fraction of incidence due to metastatic thyroid cancer. It is of great interest in our case that neoplastic pericarditis was the primary sign of papillary thyroid carcinoma with no pathologically metabolic active tissue in the thyroid gland. Few cases were reported in the literature with similar signs. Chiewvit, et al., describes a case of a 62-year-old male with a significant amount of fluid inside the pericardial cavity because of follicular carcinoma of the thyroid gland [3]. Subsequent management was the radical excision of the thyroid gland and interestingly only a small nodule was found positive for follicular carcinoma. Another case from Kovacs et al. has been described regarding a 68-year-old male who presented with pericardial effusion [4]. Cytological examination revealed metastatic papillary carcinoma. Also, Tsoukalas, et al. mentioned the rare case of pericarditis combined with an increased amount of pericardial effusion because of papillary thyroid carcinoma in a 49-year-old female patient [5] Although cases with metastatic thyroid cancer to pericardium are enormously rare, Besic, et al., reports the incidence of metastatic thyroid cancer in 45 patients [6]. More specifically, the lungs showed to reach the peak of the most common site of thyroid cancer metastasis to $78 \%$, while pericardium stood at $13 \%$ of the total cases with metastatic thyroid cancer.

Once metastatic adenocarcinoma occurs and no specific primary site of tumor like lungs, breast or intestine is found, histopathology is crucial to solve diagnostic dilemmas. Even if papillary thyroid tumors constitute the most common thyroid neoplasia, papillary carcinomas were poorly characterized by signet ring cell morphology $[7,8]$. This type of appearance is typically associated with gastric carcinomas, but it is also reported in adenocarcinomas of other primary sites [9]. The term "signet-ring" has been widely used to describe the morphology of a cell with a crescent-shaped nucleus displaced to the periphery in close proximity to the cellular membrane. This cytomorphology is secondary to the accumulation of free or vacuolated intracytoplasmic mucin, lipids, and other substances [10].

The "signet-ring" cell variant of papillary thyroid carcinoma is rare. When the first positive biopsy material is taken from an extrathyroid tissue, the differential diagnosis is very challenging. Some of the most usual primary sites for SRC carcinomas are the stomach, lungs, kidneys and less often areas of the head and neck. The SRC adenocarcinoma of the stomach should be positive for CDX2 and maybe for SATB2 [11]. The SRC adenocarcinoma of the lung should be positive for Napsin A and negative for Thyroglobulin. Metastatic melanomas are a rarer diagnosis, but we should consider it in case we observe cells negative for keratins and positive for S100 protein.

In summary, neoplastic pericardial effusion is usually found in metastatic lung cancer followed by lymphomas and rarely as a result of primary malignancy of the pericardium. Thyroid carcinomas could have distant metastasis at the initial presentation of the disease such as metastasis in lungs and pleura, bone, brain, liver and kidney. However, metastatic pericardial effusion as an initial manifestation of papillary thyroid cancer is uncommon. In such cases, histological findings could be challenging. Sporadic cases of papillary thyroid cancer with "signet-ring" cells are found in literature and as much as we know this is the first reported case of diagnosis directly from metastatic sites.

\section{References}

1. Romero-Rojas AE, Diaz-Perez JA, Mastrodimos M, Chinchilla SI. Follicular thyroid carcinoma with signet ring cell morphology: fineneedle aspiration cytology, histopathology, and immunohistochemistry. Endocr Pathol. 2013; 24: 239-245.

PubMed: https://pubmed.ncbi.nlm.nih.gov/24068558/

2. Burazor I, Imazio M, Markel G, Adler Y. Malignant Pericardial Effusion Cardiology. 2013; 124: 224-232.

PubMed: https://pubmed.ncbi.nlm.nih.gov/23571453/

3. Chiewvit S, Pusuwan P, Chiewvit P, Pleehachinda R, Attanatho V, et al. Metastatic follicular carcinoma of thyroid to pericardium. J Med Assoc Thai. 1998; 81: 799-802

PubMed: https://pubmed.ncbi.nlm.nih.gov/9803073/

4. Kovacs CS, Nguyen GK, Mullen JC, Crockford PM. Cardiac tamponade as the initial presentation of papillary thyroid carcinoma. Can J Cardiol. 1994; 10: 279-281.

PubMed: https://pubmed.ncbi.nlm.nih.gov/8143231/

5. Tsoukalas N, Kostakis ID, Demiri S, Koumakis G, Barbounis V, et al. Neoplastic pericarditis as the initial manifestation of a papillary thyroid carcinoma. Ups J Med Sci. 2013; 118: 196-198.

PubMed: https://pubmed.ncbi.nlm.nih.gov/23697667/

6. Besic N, Gazic B. Sites of metastases of anaplastic thyroid carcinoma: autopsy findings in 45 cases from a single institution. Thyroid. 2013; 23: 709-713.

PubMed: https://pubmed.ncbi.nlm.nih.gov/23148580/

7. Farhat NA, Onenerk AM, Krane JF, Dias-Santagata D, Sadow PM, et al. Primary Benign and Malignant Thyroid Neoplasms with Signet Ring Cells: Cytologic, Histologic, and Molecular Features. Am J Clin Pathol. 2017; 148: 251-258

PubMed: https://pubmed.ncbi.nlm.nih.gov/28821194/

8. Chiofalo MG, Losito NS, Fulciniti F, Setola SV, Tommaselli A, et al Axillary node metastasis from differentiated thyroid carcinoma with Hürthle and signet ring cell differentiation. A case of disseminated thyroid cancer with peculiar histologic findings. BMC Cancer. 2012; 12: 55. PubMed: https://pubmed.ncbi.nlm.nih.gov/22304797/

9. Juhlin CC, Bränström R, Shabo I, Höög A. Clear cell variant of a follicular thyroid tumor with uncertain malignant potential: a case report. Int J Surg Pathol. 2019; 27: 290-293.

PubMed: https://pubmed.ncbi.nlm.nih.gov/30370813/

10. Pagni F, Ronchi S, Di Bella C, Serra G, Costantini M, et al. Signetring cell differentiation in FNA cytology of a primitive thyroid carcinoma. Cytopathology. 2013; 24: 274-275.

PubMed: https://pubmed.ncbi.nlm.nih.gov/22409226/ 
11. Ma C, Lowenthal BM, Pai RK. SATB2 Is Superior to CDX2 in Distinguishing Signet Ring Cell Carcinoma of the Upper Gastrointestinal Tract and Lower Gastrointestinal Tract. Am J Surg Pathol. 2018; 42: 1715-1722.

PubMed: https://pubmed.ncbi.nlm.nih.gov/30212392/
12. Cameselle-Teijeiro JM, Eloy C, Sobrinho-Simões M. Pitfalls in Challenging Thyroid Tumors: Emphasis on Differential Diagnosis and Ancillary Biomarkers. Endocr Pathol. 2020; 31: 197-217. PubMed: https://pubmed.ncbi.nlm.nih.gov/32632840/ 